\title{
Use of intravitreal rituximab for treatment of vitreoretinal lymphoma
}

\author{
Kelly L Larkin, ${ }^{1}$ Ujwala S Saboo, ${ }^{2}$ Grant M Comer, ${ }^{3}$ Farzin Forooghian, ${ }^{4}$ \\ Friederike Mackensen, ${ }^{5}$ Pauline Merrill, ${ }^{6} \mathrm{H}$ Nida Sen, ${ }^{7}$ Arun Singh, ${ }_{1}^{8}$ Rohan W Essex ${ }_{1}^{9}$ \\ Stewart Lake, ${ }^{10}$ Lyndell L Lim, $^{11}$ Daniel V Vasconcelos-Santos, ${ }^{12}$ C Stephen Foster, ${ }^{2,13}$ \\ David J Wilson, ${ }^{1}$ Justine R Smith ${ }^{1,10}$
}

For numbered affiliations see end of article.

\section{Correspondence to} Professor Justine R Smith, Flinders University School of Medicine, Flinders Medical Centre Room 4E-431, Flinders Drive, Bedford Park, SA 5042, Australia;

justine.smith@flinders.edu.au

Received 19 July 2013 Revised 20 September 2013 Accepted 23 September 2013 Published Online First 24 October 2013
To cite: Larkin $\mathrm{KL}$, Saboo US, Comer GM, et al. $\mathrm{Br} J$ Ophthalmol 2014;98:99-103.

\section{ABSTRACT}

Aim Vitreoretinal lymphoma is a diffuse large B cell non-Hodgkin lymphoma. Targeting malignant cells with rituximab is being used increasingly as local chemotherapy, but information on this treatment is scant. We aimed to describe current therapeutic approaches, as well as responses to and complications of, intravitreal rituximab in patients with vitreoretinal lymphoma.

Methods Clinical data were collected in a standardised manner retrospectively on patients with vitreoretinal lymphoma treated with intravitreal rituximab.

Results 48 eyes (34 patients) with vitreoretinal lymphoma were treated with a median of 3.5 intravitreal injections of rituximab $(1 \mathrm{mg} / 0.1 \mathrm{~mL})$ for new diagnosis $(68.8 \%)$, progressive disease $(29.9 \%)$ and maintenance therapy (2.1\%). Intravitreal rituximab \pm methotrexate was the sole treatment in 19 eyes (39.6\%). 31 eyes (64.6\%) eyes achieved complete remission, after a median of 3 injections; 7 of these eyes developed recurrent disease. 11 eyes (22.9\%) achieved partial remission. Although rituximab may have contributed to complications reported in 12 eyes $(25.0 \%)$, a 2-line loss of Snellen visual acuity occurred in only 2 of those eyes (4.2\%).

Conclusions Approaches in rituximab-based intravitreal chemotherapy vary widely, but our findings suggest that this treatment may be safe and effective in inducing remission in a majority of eyes with vitreoretinal lymphoma.

\section{INTRODUCTION}

Vitreoretinal lymphoma, which represents a subset of the intraocular lymphomas, is a posterior segment tumour that typically occurs in persons aged over 60 years. ${ }^{1} 2$ In the majority of cases, vitreoretinal lymphoma is classified as a diffuse large B cell non-Hodgkin lymphoma. ${ }^{3}$ While the majority of patients present with visual disturbances, including reduced vision and floaters, most succumb to the malignancy due to extraocular central nervous system (CNS) involvement. ${ }^{4}{ }^{5} \mathrm{~A}$ definitive treatment to cure vitreoretinal lymphoma remains elusive. External-beam ocular radiotherapy $^{6}$ and/or intraocular chemotherapy ${ }^{7-9}$ have been employed by the vitreoretinal surgeons or uveitis subspecialists who frequently manage this disease, in order to achieve control locally while avoiding systemic toxicity. Intravitreally injected methotrexate alone is the most widely used form of intraocular chemotherapy, and although this approach is highly effective in inducing remission, several limitations remain with its use in vitreoretinal lymphoma. As originally described, ${ }^{10}$ the treatment schedule is intensive, involving injections biweekly during the 1 st month and weekly during the 2 nd month. The therapy may be complicated by toxic keratopathy. $^{7} \quad 8$ Finally, recurrence remains a problem for individuals who enjoy relatively prolonged survival. ${ }^{8}$ These issues have motivated a search for alternative local chemotherapeutic options for patients with vitreoretinal lymphoma.

Rituximab is a mouse-human chimeric monoclonal antibody that targets the CD20 protein on the surface of benign and malignant $\mathrm{B}$ cells; multiple cellular effects of rituximab result ultimately in the depletion of B cell populations. ${ }^{11}$ Combining rituximab with standard chemotherapy for systemic diffuse large B cell lymphoma has resulted in a substantial decrease in the mortality of this tumour. ${ }^{12}$ With rare exception, vitreoretinal malignant $\mathrm{B}$ cells express CD20. ${ }^{3}$ The first evidence that rituximab might have therapeutic benefit in vitreoretinal lymphoma came from the phase I trial of intraventricular rituximab for CNS lymphoma reported by Rubenstein $e t a l .{ }^{13}$ In this study, each of two patients with associated eye involvement experienced reduction in the intraocular tumour. Subsequently, case reports and single centre case series have described the use of intravitreal rituximab to treat up to 30 patients with vitreoretinal lymphoma. ${ }^{14-21}$

Although there is limited published experience on the use of intravitreal rituximab injections for vitreoretinal lymphoma, informal discussion with vitreoretinal surgeons and uveitis specialists indicates that this form of treatment is being employed increasingly. We compiled clinical information retrospectively and in a standardised manner for 48 eyes of 34 patients with vitreoretinal lymphoma who had been treated with intravitreal rituximab injections at 1 of 12 subspecialty ophthalmic clinics internationally. These data provide a description of the therapeutic approaches being used, as well as responses and complications for this form of treatment.

\section{METHODS}

Ophthalmologists who had treated patients with vitreoretinal lymphoma with rituximab were identified through an electronically communicated survey of the American Uveitis Society. Additional clinicians were recruited at the Association for Research in Vision and Ophthalmology 2011 Annual Meeting, after initial results of the project were presented at that meeting. For each case treated, ophthalmologists collected 
clinical data in a standardised manner retrospectively, using a data collection sheet that was approved by the Oregon Health \& Science University Institutional Review Board. One case was previously published as a case report. ${ }^{17}$

On the data collection sheet, background clinical information included: gender; race; age at presentation with lymphoma; and results of HIV testing. Description of the vitreoretinal lymphoma included: initial site of lymphoma; laterality of ocular involvement; extraocular involvement(s); method(s) of diagnosis; and previous treatment(s) of lymphoma, including focal ocular treatment (ie, ocular chemotherapy and radiotherapy) and/or extensive treatment (ie, systemic and intrathecal chemotherapy, whole brain irradiation and stem cell transplantation). Treatment with intravitreally injected rituximab was described by: dose; dosing frequency; total number of injections; duration of treatment course; other intravitreal chemotherapy and timing in relation to intravitreal rituximab injections; and other focal ocular treatment and/or extensive treatment. Measures of treatment effectiveness and safety included: induction of remission and, if applicable, number of injections to remission; and occurrence and timing of relapse; Snellen visual acuities at start and finish of treatment course; and ocular complications during or after treatment course. Duration of follow-up from 1st day of treatment was also recorded. Complete remission was defined according to criteria published by the International Primary CNS Lymphoma Collaborative Group, ${ }^{22}$ as the absence of cells in vitreous and resolution of any previously documented retinal or optic nerve infiltrates. If treatment was followed by reduction, but not disappearance, of vitreal cells and/or partial resolution of infiltrates, remission was described as partial.

\section{RESULTS}

Clinical data were collected for 34 patients who suffered from vitreoretinal lymphoma and were treated with intravitreal injection of rituximab. Of the 34 patients, 19 (55.9\%) were female and 15 (44.1\%) were male; and there were 30 (91.2\%) Caucasians, 2 (5.9\%) Asians and $1(2.9 \%)$ Hispanic. Patient age at presentation with vitreoretinal lymphoma ranged between 39 years and 86 years (median $=63$ years). None of the 19 persons $(55.9 \%)$, for whom relevant information was available, were infected with HIV. In 25 patients $(73.5 \%)$, the extraocular CNS was involved with lymphoma, and 3 of these patients had systemic involvement. Eye was the initial site of involvement in 21 patients $(61.8 \%)$. Vitreoretinal lymphoma was bilateral in 26 cases $(76.5 \%)$. Follow-up period was up to 54 months (median=11 months); at the end of the study, of the 34 subjects, 26 (76.5\%) were alive, 7 $(20.6 \%)$ were deceased and 1 (2.9\%) was lost to follow-up.

Diagnosis of vitreoretinal lymphoma was made by evaluation of the vitreous in 26 of 33 patients (76.5\%). Testing took the form of: cytology $(n=25,73.5 \%)$; immunophenotyping $(n=14$, 41.2\%); PCR for immunoglobulin $\mathrm{H}$ rearrangements $(n=10$, 29.4\%); and/or cytokine analysis, involving measurement of interleukin (IL)-10 and/or IL-6 levels ( $n=9,26.5 \%)$. One patient $(2.9 \%)$ was diagnosed with vitreoretinal lymphoma from a chorioretinal biopsy. In seven patients (20.6\%), diagnosis rested on examination of extraocular tissue, including brain in six patients and spleen in one patient. Prior to initiation of treatment with intravitreal rituximab, 23 patients (67.6\%) were treated with focal ocular treatment or extensive treatment for intraocular and/or extraocular lymphoma. Characteristics of the study cohort are presented in table 1 .

A total of 48 eyes of the 34 patients were treated with intravitreal injection(s) of rituximab for three indications: (1) new diagnosis $(n=33,68.8 \%) ;(2)$ progressive disease $(n=14$,
Table 1 Characteristics of the study cohort ( $n=34$ persons)

\begin{tabular}{lc}
\hline Variable & $\begin{array}{c}\text { Number of patients (\%) } \\
\text { or range (median) }\end{array}$ \\
\hline Female gender & $19(55.9)$ \\
Age (years) & $39-86(63)$ \\
Race or ethnicity & \\
Caucasian & $31(91.2)$ \\
Asian & $2(5.9)$ \\
Hispanic & $1(2.9)$ \\
HIV status & \\
Negative & $19(55.9)$ \\
Positive & $0(0)$ \\
Unknown & $15(44.1)$ \\
Extraocular CNS lymphoma & $25(73.5)$ \\
Bilateral ocular involvement & $26(76.5)$ \\
Method(s) of diagnosis & \\
Vitreous analysis & $26(76.5)$ \\
$\quad$ Cytology & $25(73.5)$ \\
Immunophenotyping & $14(41.2)$ \\
PCR (IgH rearrangement) & $10(29.4)$ \\
Cytokine analysis (IL-10 or IL-10:IL-6) & $9(26.5)$ \\
Chorioretinal biopsy & $1(2.9)$ \\
Extraocular tissue biopsy & $7(20.6)$ \\
Brain & $6(17.6)$ \\
Spleen & $1(2.9)$ \\
Previous treatment & \\
None & $11(32.4)$ \\
Focal ocular treatment & $11(32.4)$ \\
Other intravitreal chemotherapy* & $9(26.5)$ \\
Ocular irradiation & $4(11.8)$ \\
Extensive treatment & $20(58.8)$ \\
Systemic & $19(55.9)$ \\
Inead & $1(2.9)$ \\
Untrathecal chemothoterval (months) & $7(20.6)$ \\
\hline CNS, central nervous system; IgH, immunoglobulin H; IL, interleukin. \\
*In all cases, other intravitreal chemotherapy was with methotrexate $(0.4$ mg/0.1 mL).
\end{tabular}

29.9\%); and (3) maintenance therapy ( $n=1,2.1 \%)$. The dose used for all treatments was $1 \mathrm{mg} / 0.1 \mathrm{~mL}$. Number of injections ranged between 1 and 17 (median=4 injections). Dosing intervals ranged widely; the most common dosing frequency was monthly in 19 eyes (39.6\%). Thus, the course of treatment with intravitreally injected rituximab extended up to 15 months (median=2 months). Of the 48 eyes, 18 (37.5\%) were injected intravitreally with rituximab only; 30 eyes were injected with methotrexate $(0.4 \mathrm{mg} / 0.1 \mathrm{~mL})$. Intravitreal injections of additional medications were given on the same day in the majority of cases ( $n=26$ of 30 eyes, 86.7\%). Nineteen eyes $(39.6 \%)$ received no treatment other than intravitreally injected chemotherapy; for 29 eyes (60.4\%), patients were also treated with extensive chemotherapy (as defined above). Intravitreal rituximab treatment is described in table 2.

Of the 48 treated eyes, $31(64.6 \%)$ demonstrated complete remission, $11(22.9 \%)$ demonstrated partial remission; and 4 $(8.3 \%)$ had no response. For two eyes $(4.2 \%)$, remission could 
not be judged because they were clinically free of lymphoma when treatment was initiated; for one eye, lymphoma was in remission and treatment was given to maintain remission, and for one eye, the diagnostic vitrectomy resulted in clinical clearing of vitreous cells. For the 31 eyes that demonstrated complete remission, clearing of vitreous cells or retinal or optic nerve infiltrates occurred following a median of three injections (range $=1-10$ injections). Of these 31 eyes, 10 (32.3\%) were treated with only intravitreal rituximab \pm methotrexate. Of the 11 eyes in partial remission, $2(18.1 \%)$ were treated with only intravitreal rituximab \pm methotrexate. Seven of the 31 eyes that achieved complete remission developed recurrent disease between 5 months and 26 months (median=17.5 months) from the date of first injection.

Complications were reported for 15 (31.3\%) of the 48 treated eyes. These complications were classified as either potentially related to the intravitreal rituximab injection, or clearly unrelated to the therapy. The former group included: cataract in nine eyes $(18.8 \%)$; elevated intraocular pressure in two eyes $(4.2 \%)$; granulomatous anterior uveitis in one eye $(2.1 \%)$; vitreous haemorrhage in one eye $(2.1 \%)$; and rhegmatogenous retinal detachment in one eye $(2.1 \%)$. Eight of the nine eyes with cataract and the eye with retinal detachment had undergone vitrectomy. Complications that were observed, but had causes other than intravitreal rituximab injection, included:

Table 2 Description of intravitreal rituximab treatment $(n=48$ eyes)

\begin{tabular}{|c|c|}
\hline Variable & $\begin{array}{l}\text { Number of eyes }(\%) \\
\text { or range (median) }\end{array}$ \\
\hline \multicolumn{2}{|l|}{ Indication for treatment } \\
\hline New diagnosis & $33(68.8)$ \\
\hline Progressive disease & $14(29.2)$ \\
\hline Maintenance therapy & $1(2.1)$ \\
\hline \multicolumn{2}{|l|}{ Dose } \\
\hline $1 \mathrm{mg} / 0.1 \mathrm{~mL}$ & $48(100)$ \\
\hline \multicolumn{2}{|l|}{ Dosing frequency } \\
\hline Single treatment & $4(8.3)$ \\
\hline Weekly & $2(4.2)$ \\
\hline 2-Weekly & $10(20.8)$ \\
\hline Monthly & 19 (39.6) \\
\hline 6-Weekly & $1(2.1)$ \\
\hline 2-Monthly & $1(2.1)$ \\
\hline Variable & $11(22.9)$ \\
\hline Total number of injections & $1-17(3.5)$ \\
\hline Duration of treatment (months) & $<1-15(2)$ \\
\hline \multicolumn{2}{|l|}{ Other treatment } \\
\hline None & $8(16.7)$ \\
\hline Focal ocular alone & $11(22.9)$ \\
\hline Other intravitreal chemotherapy* & $11(22.9)$ \\
\hline Extensive treatment alone & $9(18.8)$ \\
\hline Systemic chemotherapy & $8(16.7)$ \\
\hline Stem cell transplantation & $1(2.1)$ \\
\hline Focal ocular and extensive treatment & $20(41.7)$ \\
\hline Other intravitreal+systemic chemotherapy & $15(31.2)$ \\
\hline Other intravitreal+intrathecal chemotherapy & $2(4.2)$ \\
\hline Other treatment combinations $†$ & $3(6.3)$ \\
\hline \multicolumn{2}{|c|}{$\begin{array}{l}\text { *In all cases, intravitreal chemotherapy was with methotrexate }(0.4 \mathrm{mg} / 0.1 \mathrm{~mL}) \text {. } \\
\text { tOther combinations of focal ocular and extensive treatment included: other } \\
\text { intravitreal+systemic chemotherapy+whole brain irradiation }(n=1) \text {; ocular irradiation } \\
\text { +intrathecal chemotherapy }(n=1) \text {; other intravitreal+intrathecal+systemic } \\
\text { chemotherapy+whole brain irradiation }(n=1) \text {. }\end{array}$} \\
\hline
\end{tabular}

methotrexate-related keratopathy ${ }^{8}$ in two eyes (4.2\%); bloodbrain barrier disruption-associated maculopathy ${ }^{23}$ in two eyes (4.2\%); secondary lymphomatous glaucoma in one eye $(2.1 \%)$; choroidal neovascular membrane at the site of an old chorioretinal scar in one eye (2.1\%); and lymphomatous exudative retinal detachment in one eye $(2.1 \%)$. Outcomes of intravitreal rituximab treatment, including complications, are summarised in table 3.

Five of the injected 48 eyes experienced significant loss of vision, defined as loss of two or more lines of Snellen visual acuity. In three cases, loss of vision was clearly caused by pathologies that were unrelated to intravitreal injection of rituximab: (1) blood-brain barrier disruption-associated maculopathy; (2) lymphomatous exudative retinal detachment; and (3) choroidal neovascular membrane at the site of an old chorioretinal scar. In the other two cases, intravitreal injection of rituximab may have been responsible for vision loss, although both eyes had also undergone vitrectomy, which may also have been causative: (4) cataract; and (5) rhegmatogenous retinal detachment. Snellen fraction visual acuities pre-intravitreal and post-intravitreal rituximab treatment are shown graphically in figure 1 .

\section{DISCUSSION}

Intravitreally delivered rituximab is being employed increasingly as a form of local chemotherapy for vitreoretinal lymphoma. On the other hand, there is a paucity of literature relating to effectiveness and safety of this approach, as well as the optimal treatment regimen. By drawing on experience of 12 subspecialty clinics internationally, and collecting information relating to 34 patients (and 48 eyes) with vitreoretinal lymphoma in standardised format, we are able to present a unique overview of this treatment modality. In our study, the most common indication

Table 3 Outcomes of intravitreal rituximab treatment

\begin{tabular}{lc}
\hline Variable & $\begin{array}{c}\text { Number of eyes (\%) } \\
\text { or range (median) }\end{array}$ \\
\hline $\begin{array}{l}\text { Treated eyes ( } \mathrm{n}=48 \text { ) } \\
\text { Remission }\end{array}$ \\
$\begin{array}{l}\text { Complete } \\
\text { Partial }\end{array}$ \\
None & $31(64.6)$ \\
Not applicable & $11(22.9)$ \\
Complications & $4(8.3)$ \\
Related to treatment (or unable to exclude & $2(4.2)$ \\
treatment as a cause) & $12(25.0)$ \\
$\quad$ Cataract & \\
$\quad$ Elevated intraocular pressure & $9(18.8)$ \\
$\quad$ Anterior uveitis & $2(4.2)$ \\
$\quad$ Vitreous haemorrhage & $1(2.1)$ \\
$\quad$ Retinal detachment & $1(2.1)$ \\
$\quad$ None & $1(2.1)$ \\
Not related to treatment & $36(75.0)$ \\
$\quad$ Keratopathy & $6(12.5)$ \\
Maculopathy & $2(4.2)$ \\
Glaucoma & $2(4.2)$ \\
Choroidal neovascularisation & $1(2.1)$ \\
Retinal detachment & $1(2.1)$ \\
Eyes with complete remission ( $n=31$ ) & $1(2.1)$ \\
Number of injections to remission & $1-10(3)$ \\
Relapse & $6(19.4)$ \\
Time to relapse (months from day of first treatment) & $5-26(17.5)$ \\
\hline
\end{tabular}




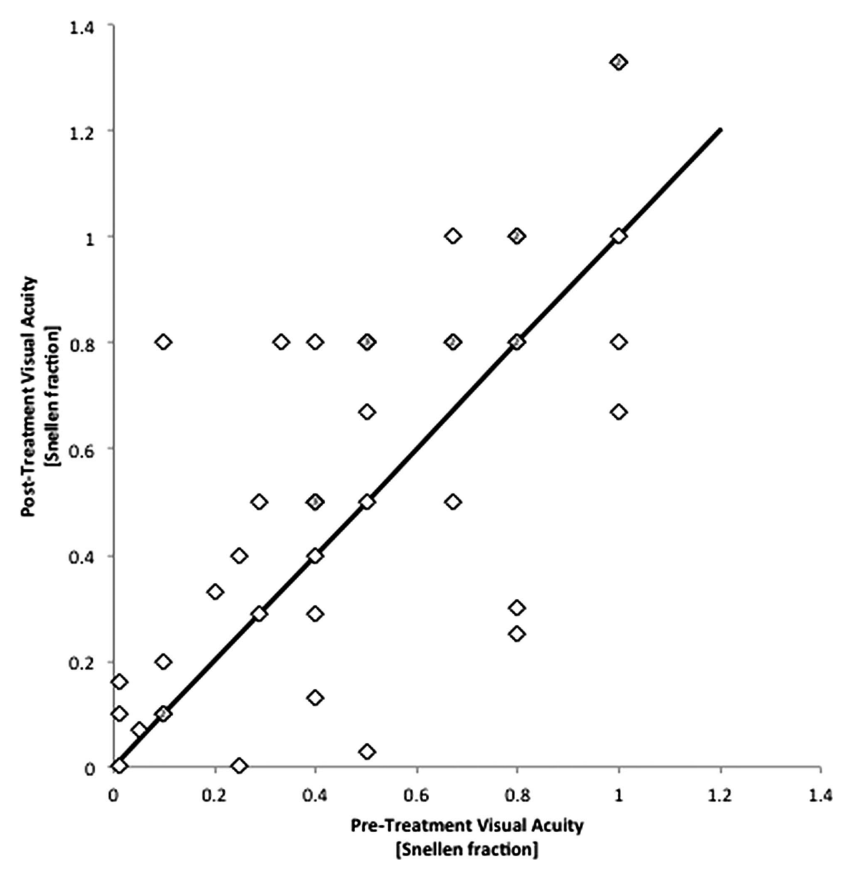

Figure 1 Graph showing Snellen visual acuity fraction for 42 eyes with vitreoretinal lymphoma, pre-treatment and post-treatment with intravitreal rituximab. For six eyes, pre-treatment and/or post-treatment visual acuities could not be quantified or were not recorded; one of these eyes had loss of visual acuity exceeding two Snellen lines.

for intravitreal rituximab injection was newly diagnosed tumour, but the treatment was also employed in cases of progressive disease and to maintain remission. Courses of intravitreal rituximab (median of 3.5 injections of $1 \mathrm{mg} / 0.1 \mathrm{~mL}$ rituximab per eye) were administered alone, or in combination with other local and/or systemic chemotherapeutic and radiotherapeutic approaches. Approximately two-thirds of eyes were treated concurrently with intravitreal methotrexate. Remission was observed in over $90 \%$ of the 46 treated eyes in which response could be judged, and was complete in the two-thirds of these. Complications were observed in approximately a third of treated eyes, and two eyes suffered significant visual acuity loss that may have been related to treatment.

In our study, $31(64.6 \%)$ of 48 lymphomatous eyes treated with intravitreal rituximab demonstrated a complete remission. The use of extraocular treatments often complicates interpretation of the effectiveness of local therapies for vitreoretinal lymphoma. Our series included 15 patients who received no treatment other than intravitreal rituximab \pm methotrexate; 9 $(60 \%)$ of these patients had complete remission, representing a similar percentage to that recorded for the entire group. The literature contains reports of up to 30 patients with vitreoretinal lymphoma receiving intravitreal rituximab injections. ${ }^{14-21}$ Effectiveness of the treatment is not documented for all 30 cases, but cases of non-responsiveness are not described. In the largest case series to date, Hashida et al, ${ }^{19}$ described the treatment of 20 eyes of 13 patients with isolated vitreoretinal lymphoma by four weekly injections of intravitreal rituximab; all patients experienced clinical benefit. Importantly, in presenting results for a relatively large group of patients, our study identified cases that did not respond $(8.3 \%)$ or responded partially $(22.9 \%)$ to the treatment. Seven $(22.6 \%)$ of the 31 eyes that were judged to respond completely to intravitreal rituximab treatment developed recurrent disease an average of 17.5 months from the date of first injection. Thus, the intravitreal rituximab-based protocols were not superior to intravitreal methotrexate alone in terms of preventing relapse.

Habot-Wilner et al, ${ }^{24}$ observed no clinical, histological or functional ill-effects up to 4 weeks after the intravitreal injection of rituximab $(0.1 \mathrm{mg} / 1 \mathrm{~mL})$ in the rabbit, but expression of glial fibrillary acidic protein by Müller cells suggested retinal stress. To be as conservative as possible in evaluating the safety of the new therapy, we recorded any complications observed during the course of intravitreal rituximab. Twenty-five per cent of 48 eyes suffered complications that may have been related to this treatment. Significant vision loss was recorded for two of these eyes. Cataract was by far the most common complication, affecting $18.8 \%$ of eyes. It is uncertain if intravitreal rituximab injections contributed to the development or progression of cataract, since most patients with vitreoretinal lymphoma were of a consistent age for the condition. In addition, eight of the nine eyes with cataract had been treated with vitrectomy, which is well known to cause this complication. ${ }^{25}$ One patient suffered a retinal detachment secondary to a retinal tear that might have been caused by intravitreal injection of rituximab, but was likely caused by vitrectomy, being the more invasive procedure. Two cases of raised intraocular pressure and a case of granulomatous anterior uveitis were more clearly related to the intravitreal rituximab treatments. In the series reported by Hashida et al, ${ }^{19}$ both these complications were described, albeit at higher frequency. One vitreous haemorrhage was observed and is a recognised complication of intravitreal injection. ${ }^{26}$ In addition, $12.5 \%$ of 48 eyes suffered complications that were clearly related to the disease or other aspects of therapy, including methotrexate-related keratopathy. Notably, keratopathy was relatively uncommon among our patients, when compared with patients treated with standard methotrexate protocols (ie, $4.2 \%$ vs $58-100 \%{ }^{7-9}$ ).

Rituximab was delivered intravitreally in a dose of $1 \mathrm{mg}$ / $0.1 \mathrm{~mL}$ for all our cases. Selection of this dose related in part to convenience, since the medication was commercially available for systemic administration at a concentration of $10 \mathrm{mg} / \mathrm{mL}$. However, the dose was also consistent with results of preclinical evaluations. Based on their studies conducted in the New Zealand white rabbit, Kim et $a^{27}$ estimated that the intravitreal concentration of rituximab would remain over $10 \mathrm{ng} / \mathrm{mL}$ for 72 days after intravitreal injection of $1 \mathrm{mg} / 0.1 \mathrm{~mL}$; unrelated work in Dutch-belted rabbits showed that the same treatment resulted in deposition of drug through the entire thickness of retina. ${ }^{28}$ Mice transplanted intraocularly with human CD20-transfected mouse lymphoma cells, to simulate human vitreoretinal lymphoma, experienced significantly delayed progression or cure of tumour following intravitreal injection of $5 \mu \mathrm{g} / 0.5 \mu \mathrm{L}$ rituximab. ${ }^{29}$

While the dose of rituximab was uniform, there was considerable variation in the frequency at which the drug was administered to our patients, as well as the total number of treatments that were given. This wide variation is also found in previous reports that provide a dosing schedule. ${ }^{14-17} 1920$ Reported dosing intervals include single injections, multiple injections given at variable intervals, and multiple injections given at fixed intervals ranging from weekly to monthly. The number of treatments varies between 1 and 18 injections per eye. While lack of standardisation to a treatment schedule is not unexpected at the introduction of new therapy, it complicates interpretation of the clinical experience and therefore is an important area for future study. A majority of eyes (62.5\%) in our series were treated with the combination of rituximab and methotrexate. In treatment of other forms of diffuse large B cell lymphoma, rituximab is combined with non-biological chemotherapeutic agents as 
standard. ${ }^{12}$ Extrapolating the principle to intraocular chemotherapy supports using a combination of the two drugs.

In summary, our study describes clinical outcomes of intravitreal rituximab chemotherapy in patients with vitreoretinal lymphoma. Common to other studies on this rare condition, our conclusions are limited by the small number of subjects and the retrospective nature of the data collection. However, our patient cohort is the largest reported to date in any study of local chemotherapy for vitreoretinal lymphoma. Additionally, data collection was standardised across all cases. Our data suggest that intravitreal rituximab is a safe therapeutic approach for vitreoretinal lymphoma. They also support the effectiveness of this therapy, but not in all cases as has been suggested by previous reports with smaller numbers of subjects. Although not superior to single-agent intraocular chemotherapy with methotrexate in terms of preventing tumour recurrences, the number of injections and the occurrence of keratopathy were reduced in our rituximab-based treatment protocols, in comparison with those reported for protocols using methotrexate alone. ${ }^{78}$

A major strength of our work is its multicentre nature, which has provided an overview of therapeutic approaches across multiple clinical practices. An important message is that there is considerable variation in intravitreal rituximab protocols between centres. This factor makes it impossible to recommend an optimal treatment schedule. The rare occurrence of the tumour and ongoing medical advances for treatment of systemic B cell lymphoma, which are likely to be considered for vitreoretinal lymphoma, create difficulties for evaluating any treatment, even by multicentre randomised controlled trial. Thus, while uncertainty remains regarding an optimal rituximab-based approach, a new antihuman CD20 antibody with enhanced affinity for CD16and superior tumoricidal activity in the mouse model of vitreoretinal lymphoma-is under consideration for Phase I/II clinical trials. ${ }^{30}$ Since results of any multicentre clinical trial may be outdated before coming to press, a more successful approach to evaluating new and established treatments of vitreoretinal lymphoma may be an international registry.

\section{Author affiliations \\ ${ }_{1}^{1}$ Casey Eye Institute, Oregon Health \& Science University, Portland, Oregon, USA ${ }^{2}$ Massachusetts Eye Research and Surgery Institution, and Ocular Immunology and Uveitis Foundation, Cambridge, Massachusetts, USA \\ ${ }^{3}$ Kellogg Eye Center, University of Michigan, Ann Arbor, Michigan, USA \\ ${ }^{4}$ Department of Ophthalmology and Visual Sciences, University of British Columbia, Vancouver, British Columbia, Canada \\ ${ }^{5}$ Interdisciplinary Uveitis Centre, Department of Ophthalmology, Universitätsklinikum Heidelberg, Heidelberg, Germany \\ ${ }^{6}$ Department of Ophthalmology, Rush University, Chicago, Illinois, USA \\ ${ }^{7}$ National Eye Institute, National Institutes of Health, Bethesda, Maryland, USA \\ ${ }^{8}$ Cleveland Clinic, Cleveland, Ohio, USA \\ ${ }^{9}$ Department of Ophthalmology, Australian National University, Canberra, Australia \\ ${ }^{10}$ Clinical \& Molecular Medicine, Flinders University, Adelaide, Australia \\ ${ }^{11}$ Centre for Eye Research Australia, University of Melbourne, Melbourne, Australia \\ ${ }^{12}$ Department of Uveitis and Ophthalmic Pathology, Universidade Federal de Minas Gerais, Belo Horizonte, Brazil \\ ${ }^{13}$ Department of Ophthalmology, Harvard University, Cambridge, Massachusetts, USA}

Acknowledgements The authors wish to thank Liam Ashander, BS, for assistance in the manuscript preparation.

Contributors . KLL and JRS: Design, Data interpretation, Manuscript draft. GMC, USS, FF, FM, PM, HNS, AS, RWE, SL, LLL, DVV-S, CSF and DJW: Analysis/Collection of data, Manuscript revision. All authors give final approval of the version to be published.

Funding This work was supported in part by Research to Prevent Blindness (Unrestricted Grant to: Casey Eye Institute, Oregon Health \& Science University).

Competing interests None.

Ethics approval Oregon Health \& Science University Institutional Review Board.

Provenance and peer review Not commissioned; externally peer reviewed.

\section{REFERENCES}

1 Chan CC, Rubenstein JL, Coupland SE, et al. Primary vitreoretinal lymphoma: a report from an International Primary Central Nervous System Lymphoma Collaborative Group symposium. Oncologist 2011;16:1589-99.

2 Davis JL. Intraocular lymphoma: a clinical perspective. Eye 2013;27:153-62.

3 Coupland SE, Heimann H, Bechrakis NE. Primary intraocular lymphoma: a review of the clinical, histopathological and molecular biological features. Graefes Arch Clin Exp Ophthalmol 2004;242:901-13.

4 Grimm SA, McCannel CA, Omuro AM, et al. Primary CNS lymphoma with intraocular involvement: International PCNSL Collaborative Group Report. Neurology 2008;71: 1355-60.

5 Grimm SA, Pulido JS, Jahnke K, et al. Primary intraocular lymphoma: an International Primary Central Nervous System Lymphoma Collaborative Group Report. Ann Oncol 2007;18:1851-5.

6 Berenbom A, Davila RM, Lin HS, et al. Treatment outcomes for primary intraocular lymphoma: implications for external beam radiotherapy. Eye 2007;21:1198-201.

7 Frenkel $\mathrm{S}$, Hendler $\mathrm{K}$, Siegal T, et al. Intravitreal methotrexate for treating vitreoretinal lymphoma: 10 years of experience. Br J Ophthalmol 2008;92:383-8.

8 Smith JR, Rosenbaum JT, Wilson DJ, et al. Role of intravitreal methotrexate in the management of primary central nervous system lymphoma with ocular involvement. Ophthalmology 2002;109:1709-16.

9 Sou R, Ohguro N, Maeda T, et al. Treatment of primary intraocular lymphoma with intravitreal methotrexate. Jpn J Ophthalmol 2008;52:167-74.

10 Fishburne BC, Wilson DJ, Rosenbaum JT, et al. Intravitreal methotrexate as an adjunctive treatment of intraocular lymphoma. Arch Ophthalmol 1997;115: 1152-6.

11 Eisenberg R, Looney RJ. The therapeutic potential of anti-CD20 "what do B-cells do?". Clin Immunol 2005;117:207-13.

12 Molina A. A decade of rituximab: improving survival outcomes in non-Hodgkin's lymphoma. Annu Rev Med 2008;59:237-250.

13 Rubenstein JL, Fridlyand J, Abrey L, et al. Phase I study of intraventricular administration of rituximab in patients with recurrent CNS and intraocular lymphoma. J Clin Oncol 2007;25:1350-6.

14 Kitzmann AS, Pulido JS, Mohney BG, et al. Intraocular use of rituximab. Eye 2007;21:1524-7.

15 Ohguro N, Hashida N, Tano Y. Effect of intravitreous rituximab injections in patients with recurrent ocular lesions associated with central nervous system lymphoma. Arch Ophthalmol 2008;126:1002-3.

16 Itty S, Olson JH, O'Connell DJ, et al. Treatment of primary intraocular lymphoma (PIOL) has involved systemic, intravitreal or intrathecal chemotherapy and/or radiotherapy. Retina 2009;29:415-6.

17 Yeh S, Wilson DJ. Combination intravitreal rituximab and methotrexate for massive subretinal lymphoma. Eye 2010;24:1625-7.

18 Turaka K, Bryan JS, De Souza S, et al. Vitreoretinal lymphoma: changing trends in diagnosis and local treatment modalities at a single institution. Clin Lymphoma Myeloma Leuk 2012;12:412-17.

19 Hashida N, Ohguro N, Nishida K. Efficacy and complications of intravitreal rituximab injection for treating primary vitreoretinal lymphoma. Trans Vis Sci Tech 2012;1: http:doi:1110.1167/tvst.1161.1163.1161.

20 Raja H, Snyder MR, Johnston PB, et al. Effect of intravitreal methotrexate and rituximab on interleukin-10 levels in aqueous humor of treated eyes with vitreoretinal lymphoma. PloS one 2013;8:e65627.

21 Levasseur SD, Wittenberg LA, White VA. Vitreoretinal lymphoma: a 20-year review of incidence, clinical and cytologic features, treatment, and outcomes. JAMA Ophthalmology 2013;131:50-5.

22 Abrey LE, Batchelor TT, Ferreri AJ, et al. Report of an international workshop to standardize baseline evaluation and response criteria for primary CNS lymphoma. J Clin Oncol 2005;23:5034-43.

23 Vicuna-Kojchen J, Frenkel S, Siegal T, et al. Maculopathy in patients with primary CNS lymphoma treated with chemotherapy in conjunction with blood-brain barrier disruption. Br J Ophthalmol 2008;92:231-5.

24 Habot-Wilner Z, Shahar J, Zemel $E$, et al. Retinal toxicity of intravitreal rituximab in albino rabbits. Retina 2013;33:649-56.

25 Recchia FM, Scott IU, Brown GC, et al. Small-gauge pars plana vitrectomy: a report by the American Academy of Ophthalmology. Ophthalmology 2010;117:1851-7.

26 Jager RD, Aiello LP, Patel SC, et al. Risks of intravitreous injection: a comprehensive review. Retina 2004;24:676-98.

$27 \mathrm{Kim} \mathrm{H}, \mathrm{Csaky} \mathrm{KG}$, Chan CC, et al. The pharmacokinetics of rituximab following an intravitreal injection. Exp Eye Res 2006;82:760-6.

28 Pulido JS, Bakri SJ, Valyi-Nagy T, et al. Rituximab penetrates full-thickness retina in contrast to tissue plasminogen activator control. Retina 2007;27:1071-3.

29 Mineo JF, Scheffer A, Karkoutly C, et al. Using human CD20-transfected murine lymphomatous B cells to evaluate the efficacy of intravitreal and intracerebral rituximab injections in mice. Invest Ophthalmol Vis Sci 2008;49:4738-45.

30 Ben Abdelwahed R, Donnou S, Ouakrim H, et al. Preclinical study of a glycoengineered anti-human CD20 antibody in murine models of primary cerebral and intraocular B-cell lymphomas. Invest Ophthalmol Vis Sci 2013;54:3657-65. 


\section{Use of intravitreal rituximab for treatment of vitreoretinal lymphoma}

Kelly L Larkin, Ujwala S Saboo, Grant M Comer, et al.

Br J Ophthalmol 2014 98: 99-103 originally published online October 24, 2013

doi: 10.1136/bjophthalmol-2013-304043

Updated information and services can be found at:

http://bjo.bmj.com/content/98/1/99.full.html

These include:

References This article cites 29 articles, 8 of which can be accessed free at: http://bjo.bmj.com/content/98/1/99.full.html\#ref-list-1

Email alerting Receive free email alerts when new articles cite this article. Sign up in service the box at the top right corner of the online article.

Notes

To request permissions go to:

http://group.bmj.com/group/rights-licensing/permissions

To order reprints go to:

http://journals.bmj.com/cgi/reprintform

To subscribe to BMJ go to:

http://group.bmj.com/subscribe/ 